\title{
Cosmic-ray propagation in molecular clouds
}

\author{
Marco Padovani and Daniele Galli
}

\begin{abstract}
Cosmic-rays constitute the main ionising and heating agent in dense, starless, molecular cloud cores. We reexamine the physical quantities necessary to determine the cosmic-ray ionisation rate (especially the cosmic ray spectrum at $E<1 \mathrm{GeV}$ and the ionisation cross sections), and calculate the ionisation rate as a function of the column density of molecular hydrogen. Available data support the existence of a low-energy component (below $\sim 100 \mathrm{MeV}$ ) of cosmic-ray electrons or protons responsible for the ionisation of diffuse and dense clouds. We also compute the attenuation of the cosmic-ray flux rate in a cloud core taking into account magnetic focusing and magnetic mirroring, following the propagation of cosmic rays along flux tubes enclosing different amount of mass and mass-to-flux ratios. We find that mirroring always dominates over focusing, implying a reduction of the cosmic-ray ionisation rate by a factor of $3-4$ depending on the position inside the core and the magnetisation of the core.
\end{abstract}

\section{Introduction}

Although the origin of the cosmic radiation and the mechanisms of acceleration are not well established, this corpuscular radiation has a central role in the high-energy processes of our galaxy. For instance, cosmic rays (CRs) approximately constitute one third of the energy density of the interstellar

M. Padovani

Laboratoire de Radioastronomie Millimétrique, UMR 8112 du CNRS, École Normale Supérieure et Observatoire de Paris, 24 rue Lhomond, 75231 Paris cedex 05, France e-mail: padovanielra.ens.fr

D. Galli

INAF - Osservatorio Astrofisico di Arcetri, Largo E. Fermi 5, 50125 Firenze, Italy e-mail: galliearcetri.astro.it 
medium and, on a galactic scale, they form a relativistic gas whose pressure is comparable with that of the galactic magnetic field. CR electrons are a source of bremßtrahlung, synchrotron emission and inverse Compton scattering, revealing the large-scale configuration and the intensity of the galactic magnetic field. CR nuclei are the unique sample of interstellar matter which is measurable out of the solar system, including all the elements, from hydrogen to actinides. The abundances of elements and isotopes of CR nuclei bring information not only with respect to their origin, but, through the radioactive species, show the required time for their acceleration and the history of their propagation in the interstellar magnetic field.

Low-energy CRs are the dominant source of ionisation for molecular cloud cores and they represent an important source of heating because the energy of primary and secondary electrons produced by the ionisation process is in large part converted into heat by inelastic collisions with ISM atoms and molecules. In addition, the observed diffuse gamma-ray emission from the galactic plane is believed to be the result of the decay of neutral pions produced during inelastic collisions of high-energy $(>1 \mathrm{GeV})$ CRs with the ISM 18.

In general, the $\mathrm{CR}$ ionisation rate in the interstellar gas depends on the relative amount of $\mathrm{H}, \mathrm{H}_{2}$, and $\mathrm{He}$ [13. The first theoretical determination of the $\mathrm{CR}$ ionisation rate was performed for clouds made only by atomic hydrogen by Hayakawa et al. 27]. They assumed a proton specific intensity (hereafter, for simplicity, spectrum) proportional to the proton energy $E_{\mathrm{p}}$ for $0.1 \mathrm{MeV}<E_{\mathrm{p}}<10 \mathrm{MeV}$ and computed $\zeta^{\mathrm{H}} \approx 4 \times 10^{-16} \mathrm{~s}^{-1}$. Spitzer \& Tomasko 52 determined a value (actually a lower limit) of $\zeta^{\mathrm{H}} \gtrsim 6.8 \times 10^{-18} \mathrm{~s}^{-1}$ for $\mathrm{H}$ I clouds, assuming a $\mathrm{CR}$ proton spectrum declining below $E_{\mathrm{p}} \approx 50 \mathrm{MeV}$, and an upper limit of $\zeta^{\mathrm{H}} \lesssim 1.2 \times 10^{-15} \mathrm{~s}^{-1}$, taking into account an additional flux of $\sim 2 \mathrm{MeV}$ protons produced by supernova explosions. To obtain the $\mathrm{CR}$ ionisation rate of molecular hydrogen, $\zeta^{\mathrm{H}_{2}}$, a useful approximation is $1.5 \zeta^{\mathrm{H}_{2}} \approx 2.3 \zeta^{\mathrm{H}}\left[23\right.$, giving $\zeta^{\mathrm{H}_{2}} \approx 10^{-17} \mathrm{~s}^{-1}$, in agreement with the lower limit on $\zeta^{\mathrm{H}}$ of Spitzer \& Tomasko [52. This value of $\zeta^{\mathrm{H}_{2}}$ is often referred to the "standard" CR ionisation rate in molecular clouds.

A major problem in determining the CR ionisation rate is that low-energy CRs are prevented from entering the heliosphere by the solar wind and the interplanetary magnetic field. In fact, the outer part of the solar atmosphere, namely the solar corona, is in continuous expansion, producing a plasma flux towards the interplanetary medium: the solar wind. The solar magnetic field is frozen with the plasma and radially dragged outwards, and this field strongly affects the extrasolar CR particles. In particular, less energetic particles are swept away from the solar system and for this reason, the extrasolar CR density we observe from the Earth is lower than the density of the local interstellar medium and the observed spectra are modulated, that is altered with respect to their original shapes. In practice, this means that Earth-based measurements of CR fluxes give no information on the interstellar spectrum of protons and heavy nuclei for energies below $\sim 1 \mathrm{GeV} /$ nucleon. Solar modu- 
lation also suppresses the flux of low-energy CR electrons, that already shows considerable fluctuations at energies of $10-100 \mathrm{GeV}$ [4. Since the cross section for ionisation of molecular hydrogen by collisions with protons and electrons has a maximum at $\sim 10 \mathrm{keV}$ and $\sim 50 \mathrm{eV}$, respectively (see Sect. 2), it is clear that a knowledge of $\mathrm{CR}$ spectrum at low energies is an important limiting factor for any accurate calculation of the ionisation rate in the ISM. A direct measurement of the shape of the CR spectrum at these energies will be possible only when spacecrafts such as Pioneer and Voyager are well beyond the heliopause, the outermost boundary for solar modulation effects, believed to lie at 100-150 AU from the Sun.

Over the past three decades, several values of $\zeta^{\mathrm{H}}$ ranging from a few $10^{-17} \mathrm{~s}^{-1}$ to a few $10^{-16} \mathrm{~s}^{-1}$ have been obtained in diffuse interstellar clouds from measurements of the abundances of various chemical species, in particular $\mathrm{OH}$ 1, 25, 2] and HD [59, 17. However, the derived rates are sensitive to several model assumptions, e.g. the value of specific chemical reaction rates and the intensity of the UV background. In dense molecular clouds, determining the CR ionisation rate is made even more uncertain by the sensitivity of molecular abundances to the level of depletion of the various species and the role of small and large grains in the chemical network. The values of $\zeta^{\mathrm{H}_{2}}$ derived by Caselli et al. [5] through $\mathrm{DCO}^{+}$and $\mathrm{HCO}^{+}$abundance ratios span a range of about two orders of magnitudes from $\sim 10^{-17} \mathrm{~s}^{-1}$ to $\sim 10^{-15} \mathrm{~s}^{-1}$, with a scatter that may in part reflect intrinsic variations of the CR flux from core to core. Finally, values of $\zeta^{\mathrm{H}_{2}}$ of a few times $10^{-17} \mathrm{~s}^{-1}$ have been obtained in clouds of higher column density $\left(N\left(\mathrm{H}_{2}\right) \sim 10^{23}-10^{24} \mathrm{~cm}^{-2}\right)$ like the envelopes surrounding massive protostellar sources [57, 16].

The discovery of significant abundances of $\mathrm{H}_{3}^{+}$in diffuse clouds 37, confirmed by follow-up detections [20, 38, 32, has led to values of $\zeta^{\mathrm{H}_{2}}$ larger by about one order of magnitude than both the "standard" rate and previous estimates based on the abundance of $\mathrm{OH}$ and $\mathrm{HD}$ in dense clouds. Given the relative simplicity of the chemistry of $\mathrm{H}_{3}^{+}$, it is now believed that diffuse clouds are characterised by $\mathrm{CR}$ ionisation rates $\zeta^{\mathrm{H}_{2}} \approx 2 \times 10^{-16} \mathrm{~s}^{-1}$ or larger. This value has been confirmed by Neufeld et al. [40] who found $\zeta^{\mathrm{H}_{2}}=0.6-2.4 \times 10^{-16} \mathrm{~s}^{-1}$ probing the $\mathrm{CR}$ ionisation rate in clouds of low $\mathrm{H}_{2}$ fraction from observations of $\mathrm{OH}^{+}$and $\mathrm{H}_{2} \mathrm{O}^{+}$.

\section{$2 \mathrm{CR}$ reactions with $\mathrm{H}_{2}$ and $\mathrm{He}$}

CR particles (electrons, protons, and heavy nuclei) impact with atoms and molecules of the ISM producing ions and electrons. Table 1 lists the main CR ionisation reactions involving $\mathrm{H}_{2}$ and $\mathrm{He}$.

In molecular clouds, a large majority of $\mathrm{CR}-\mathrm{H}_{2}$ impacts leads to the formation of $\mathrm{H}_{2}^{+}$via the ionisation reaction 
Table 1 CR reactions in molecular clouds.

$$
\begin{array}{ll}
\hline \hline p_{\mathrm{CR}}+\mathrm{H}_{2} \rightarrow p_{\mathrm{CR}}+\mathrm{H}_{2}^{+}+e & \sigma_{p}^{\text {ion. }} \\
p_{\mathrm{CR}}+\mathrm{H}_{2} \rightarrow \mathrm{H}+\mathrm{H}_{2}^{+} & \sigma_{p}^{\text {e. c. }} \\
p_{\mathrm{CR}}+\mathrm{H}_{2} \rightarrow p_{\mathrm{CR}}+\mathrm{H}+\mathrm{H}^{+}+e & \sigma_{p}^{\text {diss. ion. }} \\
p_{\mathrm{CR}}+\mathrm{H}_{2} \rightarrow p_{\mathrm{CR}}+2 \mathrm{H}^{+}+2 e & \sigma_{p}^{\text {doub. ion. }} \\
\hline e_{\mathrm{CR}}+\mathrm{H}_{2} \rightarrow e_{\mathrm{CR}}+\mathrm{H}_{2}^{+}+e & \sigma_{e}^{\text {ion. }} \\
e_{\mathrm{CR}}+\mathrm{H}_{2} \rightarrow e_{\mathrm{CR}}+\mathrm{H}+\mathrm{H}^{+}+e & \sigma_{e}^{\text {diss. ion. }} \\
e_{\mathrm{CR}}+\mathrm{H}_{2} \rightarrow e_{\mathrm{CR}}+2 \mathrm{H}^{+}+2 e & \sigma_{e}^{\text {doub. ion. }} \\
\hline p_{\mathrm{CR}}+\mathrm{He} \rightarrow p_{\mathrm{CR}}+\mathrm{He}^{+}+e & \sigma_{p}^{\text {ion. }} \\
p_{\mathrm{CR}}+\mathrm{He} \rightarrow \mathrm{H}+\mathrm{He}^{+} & \sigma_{p}^{\text {e. c. }} \\
\hline e_{\mathrm{CR}}+\mathrm{He} \rightarrow e_{\mathrm{CR}}+\mathrm{He}^{+}+e & \sigma_{e}^{\text {ion. }} \\
\hline \multicolumn{2}{c}{} \\
\multicolumn{2}{c}{k_{\mathrm{CR}}+\mathrm{H}_{2} \rightarrow k_{\mathrm{CR}}+\mathrm{H}_{2}^{+}+e,}
\end{array}
$$

where $k_{\mathrm{CR}}$ is a cosmic-ray particle of species $k$ and energy $E_{k}$, with cross section $\sigma_{k}^{\text {ion. }}$. Here we consider CR electrons $(k=e)$, protons $(k=p)$, and heavy nuclei of charge $Z e(k=Z$, with $Z \geq 2)$. Low-energy CR protons, in addition, may react with ambient $\mathrm{H}_{2}$ by electron capture reactions,

$$
p_{\mathrm{CR}}+\mathrm{H}_{2} \rightarrow \mathrm{H}+\mathrm{H}_{2}^{+}
$$

with cross section $\sigma_{p}^{\text {e. c. }}$. For an isotropic distribution of CR particles, the production rate of $\mathrm{H}_{2}^{+}$(per $\mathrm{H}_{2}$ molecule) is then

$$
\begin{aligned}
\zeta^{\mathrm{H}_{2}}= & 4 \pi \sum_{k} \int_{I\left(\mathrm{H}_{2}\right)}^{E_{\max }} j_{k}\left(E_{k}\right)\left[1+\phi_{k}\left(E_{k}\right)\right] \sigma_{k}^{\text {ion. }}\left(E_{k}\right) \mathrm{d} E_{k} \\
& +4 \pi \int_{0}^{E_{\max }} j_{p}(E) \sigma_{p}^{\text {e. c. }}\left(E_{p}\right) \mathrm{d} E_{p},
\end{aligned}
$$

where $j_{k}\left(E_{k}\right)$ is the number of CR particles of species $k$ per unit area, time, solid angle and per energy interval (hereafter, we will refer to $j_{k}\left(E_{k}\right)$ simply as the spectrum of particle $k), I\left(\mathrm{H}_{2}\right)=15.603 \mathrm{eV}$ is the ionisation potential of $\mathrm{H}_{2}$, and $E_{\max }=10 \mathrm{GeV}$ is the maximum energy considered.

The quantity $\phi_{k}\left(E_{k}\right)$ is a correction factor accounting for the ionisation of $\mathrm{H}_{2}$ by secondary electrons. In fact, secondary electrons are sufficiently energetic to induce further ionisations of $\mathrm{H}_{2}$ molecules, and their relatively short range justifies a local treatment of their ionising effects. The number of secondary ionisation produced per primary ionisation of $\mathrm{H}_{2}$ by a particle $k$ is determined by

$$
\phi_{k}\left(E_{k}\right) \equiv \frac{1}{\sigma_{k}^{\text {ion. }}\left(E_{k}\right)} \int_{I\left(\mathrm{H}_{2}\right)}^{E_{\max }^{\prime}} P\left(E_{k}, E_{e}^{\prime}\right) \sigma_{e}^{\text {ion. }}\left(E_{e}^{\prime}\right) \mathrm{d} E_{e}^{\prime},
$$


where $P\left(E_{k}, E_{e}^{\prime}\right)$ is the probability that a secondary electron of energy $E_{e}^{\prime}$ is ejected in a primary ionisation by a particle of energy $E_{k}$. The spectrum of secondary electrons declines rapidly with $E_{e}^{\prime}$ from the maximum at $E_{e}^{\prime}=0$ (Glassgold \& Langer 21]; Cecchi-Pestellini \& Aiello [8). The function $\phi_{e}\left(E_{e}\right)$ giving the number of secondary ionisations after a single ionisation by an electron of energy $E_{e}$ has been computed by Glassgold \& Langer [21] for energies of the incident electron up to $10 \mathrm{keV}$. Above a few $100 \mathrm{eV}, \phi_{e}$ increases logarithmically with $E_{e}$. For secondary electrons produced by impact of particles $k$, we adopt the scaling $\phi_{k}\left(E_{k}\right) \approx \phi_{e}\left(E_{e}=m_{e} E_{k} / m_{k}\right)$ valid in the BetheBorn approximation. Calculations by Cravens \& Dalgarno [12] confirm this scaling for protons in the range 1-100 MeV.

Additional ionisation reactions that produce electrons are the dissociative ionisation of $\mathrm{H}_{2}$ and the double ionisation of $\mathrm{H}_{2}$. These two processes contribute to the total $\mathrm{CR}$ production rate of electrons per $\mathrm{H}_{2}$ molecule,

$$
\begin{aligned}
\zeta^{e}= & 4 \pi \sum_{k} \int_{I\left(\mathrm{H}_{2}\right)}^{E_{\max }} j_{k}\left(E_{k}\right)\left[1+\phi_{k}\left(E_{k}\right)\right] \sigma_{k}^{\text {ion. }}\left(E_{k}\right) \mathrm{d} E_{k} \\
& +4 \pi \sum_{k} \int_{E^{\text {diss. ion. }}}^{E_{\max }} j_{k}(E)\left[1+\phi_{k}\left(E_{k}\right)\right] \sigma_{k}^{\text {diss. ion. }}\left(E_{k}\right) \mathrm{d} E_{k} \\
& +8 \pi \sum_{k} \int_{E^{\text {doub. ion. }}}^{E_{\max }} j_{k}\left(E_{k}\right)\left[1+\phi_{k}\left(E_{k}\right)\right] \sigma_{k}^{\text {doub. ion. }}\left(E_{k}\right) \mathrm{d} E_{k},
\end{aligned}
$$

where $E^{\text {diss. ion. }}=18.1 \mathrm{eV}$ and $E^{\text {doub. ion. }}=51 \mathrm{eV}$. The cross sections of these processes are smaller by at least one order of magnitude than the corresponding ionisation cross section, and the relative contribution of dissociative ionisation and double ionisation to the total electron production rate is expected to be small [42.

Similarly, the CR production rate of $\mathrm{He}^{+}$(per He atom) is

$$
\begin{aligned}
\zeta^{\mathrm{He}}= & 4 \pi \sum_{k} \int_{I(\mathrm{He})}^{E_{\max }} j_{k}\left(E_{k}\right)\left[1+\phi_{k}\left(E_{k}\right)\right] \sigma_{k}^{\text {ion. }}\left(E_{k}\right) \mathrm{d} E_{k} \\
& +4 \pi \int_{0}^{E_{\max }} j_{p}(E) \sigma_{p}^{\text {e. c. }}\left(E_{p}\right) \mathrm{d} E_{p}
\end{aligned}
$$

where $I(\mathrm{He})=24.587 \mathrm{eV}$ is the ionisation potential of $\mathrm{He}, \sigma_{k}^{\text {ion. }}$ is the ionisation cross sections of He for impact by particles $k$, and $\sigma_{p}^{\text {e. c. }}$ is the electron capture cross section.

Figure 1 shows that ionisation cross sections peak at about $10 \mathrm{keV}$ and $0.1 \mathrm{keV}$ for protons and electrons colliding with $\mathrm{H}_{2}$, respectively, and at about $30 \mathrm{keV}$ and $0.1 \mathrm{keV}$ for protons and electrons colliding with He, respectively. This means that, to compute reliable CR ionisation rates, the $\mathrm{CR}$ spectrum needs to be extrapolated down to $\sim \mathrm{keV}$ energies where the ionisation cross sections have their maximum. 


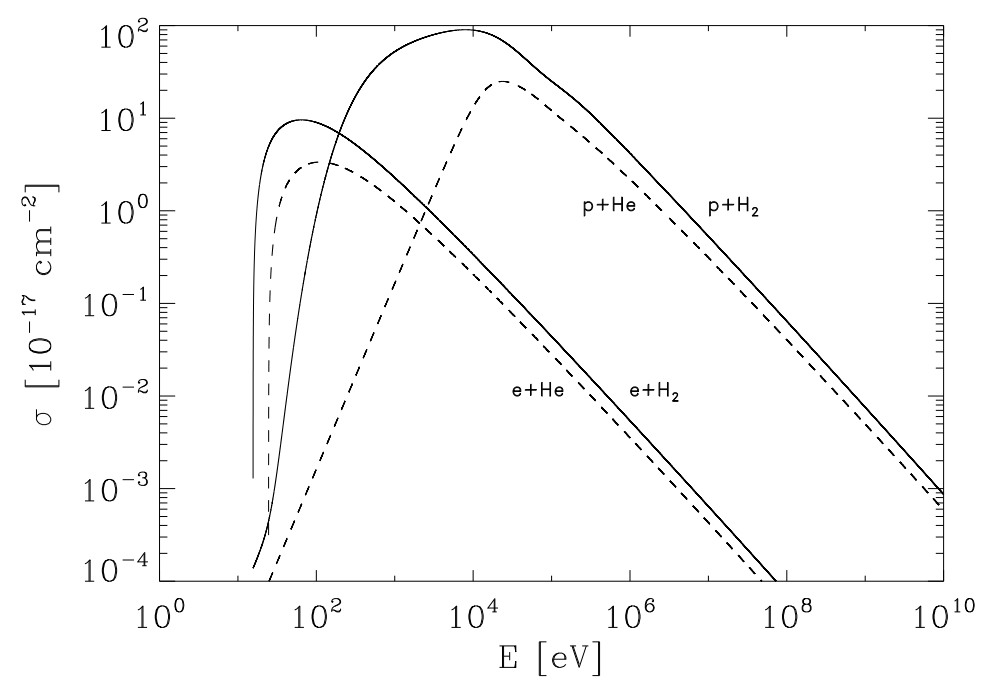

Fig. 1 Ionisation cross sections for proton and electron impact on $\mathrm{H}_{2}$ (solid lines) and on $\mathrm{He}$ (dashed lines).

\section{Local interstellar spectra}

It is generally assumed that the local interstellar (LIS) spectrum, namely the CR spectrum in the solar neighbourhood, characterises the energy distribution of CR everywhere in the galactic disk, as long as the ISM properties do not depart from the uniform conditions assumed in the propagation model.

It is very uncertain, however, whether the LIS spectrum is really representative of the whole galactic disk, especially because the Solar System resides in a low-density $\left(n \approx 10^{-3} \mathrm{~cm}^{-3}\right)$ region. We assume a uniform distribution (in space and time) of CR sources characterised by a given "source spectrum". CR propagation models can generate steady-state LIS spectra resulting from a number of processes affecting the CR transport in the galactic disk (see Sect. (4), which, in turn, can be used as input for solar modulation calculations to reproduce the $\mathrm{CR}$ spectrum and the relative abundances of $\mathrm{CR}$ particles measured at the Earth. The LIS spectra obtained in this way are clearly not uniquely defined, and a considerable range of LIS spectral shapes can be shown to be consistent with the measured CR flux with appropriate choices of parameters of the transport model (see e.g. Mewaldt et al. [36, especially their Fig. 1).

We consider for both protons and electrons a "minimum" and "maximum" LIS spectrum compatible with the available observational constraints, and we compute the resulting ionisation rates with the objective of comparing 
them with existing data for diffuse and dense clouds. We extrapolate the LIS spectra for the CR proton and electron components to lower energies with power-laws to reach the peak of the ionisation cross section. The two determinations of the proton LIS spectrum (left panel of Fig. 2) that we considered are given by Webber 61 ("minimum", hereafter W98) and Moskalenko et al. 39] ("maximum", hereafter M02). W98 estimated the LIS proton spectrum down to $\sim 10 \mathrm{MeV}$, starting from an injection spectrum parameterised as a power-law in rigidity, propagated according to the model of Webber 60. and accounting for solar modulation following Potgieter [46]. M02 reproduces the observed spectrum of protons, antiprotons, alpha nuclei, the $\mathrm{B} / \mathrm{C}$ ratio, and the diffuse $\gamma$-ray background. The two electron LIS spectra adopted (right panel of Fig. 2) both derive by Strong et al. [53]: the former ("minimum", hereafter $\mathrm{C00}$ ) is mostly derived from radio observations. It reproduces the spectrum of electrons, protons, and alpha nuclei above $\sim 10 \mathrm{GeV}$, but fails to account for the $\gamma$-ray background for photons with energies below $\sim 30 \mathrm{GeV}$ and above $\sim 1 \mathrm{GeV}$. The latter ("maximum", hereafter E00) reproduces the $\gamma$ observations at photon energies below $\sim 30 \mathrm{GeV}$ by a combination of bremßtrahlung and inverse Compton emission, assuming a steepening of the electron spectrum below $\sim 200 \mathrm{MeV}$ to compensate for the growth of ionisation losses. Our extrapolations at low energies are power-law in energy, $j(E) \propto E^{\beta}$. The values of $\beta$ are shown in Table 2

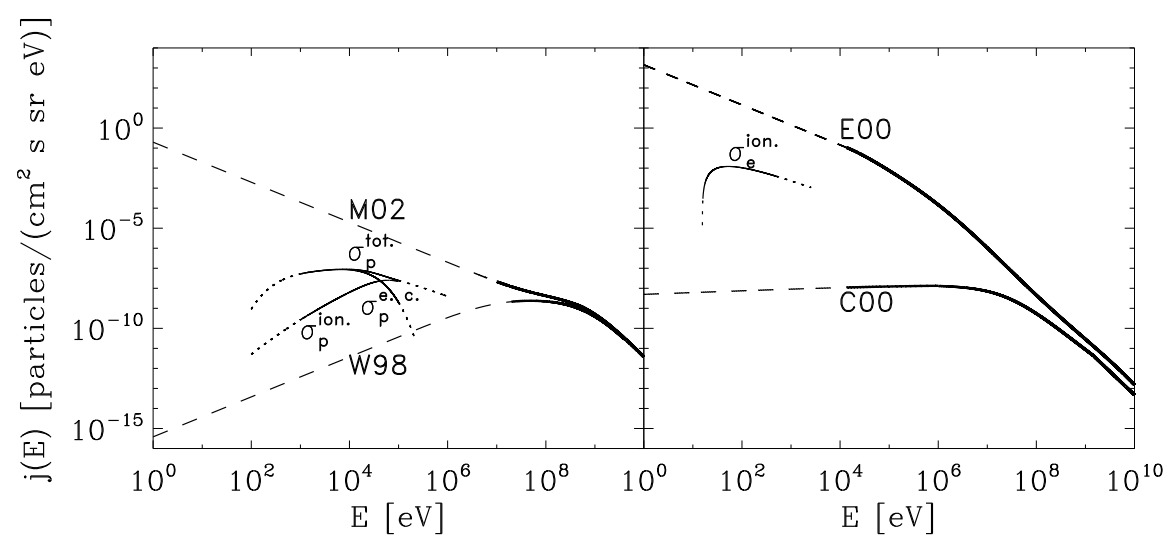

Fig. 2 Left panel: proton LIS spectra of M02 and W98 (solid curves). The dashed curves represent our power-law extrapolations of the spectra. For comparison, the cross sections for ionisation of $\mathrm{H}_{2}$ by proton impact, electron capture, and total ionisation are also shown. Right panel: electron LIS spectra of E00 and C00 (solid curves). The dashed curves represent our extrapolations of the spectra. For comparison, the cross section for ionisation of $\mathrm{H}_{2}$ by electron impact is also shown. All the cross sections are in arbitrary units. 
The values of $\zeta_{k}^{\mathrm{H}_{2}}, \zeta_{k}^{e}$ and $\zeta_{k}^{\mathrm{He}}$ per $\mathrm{H}_{2}$ molecule and He atom, respectively, obtained from numerical integration of Eq. (3), (5) and (6), with the $j_{k}\left(E_{k}\right)$ taken to be the adopted LIS spectra, are listed in Table 2 We have assumed a mixture of $\mathrm{H}_{2}$ and He with $n\left(\mathrm{H}_{2}\right)=0.5 n(\mathrm{H})$ and $n(\mathrm{He})=0.1 n(\mathrm{H})$, that is $f_{\mathrm{H}_{2}} \sim 0.83$ and $f_{\mathrm{He}} \sim 0.17$, where $f_{k}=n(k) /\left[n\left(\mathrm{H}_{2}\right)+n(\mathrm{He})\right]$, with $k=$ $\mathrm{H}_{2}$, He. We checked that our extrapolations at low energies were compatible with energy constraints. To this purpose, we computed the energy density of each CR component, defined as

$$
\mathcal{E}_{k}=4 \pi \int_{0}^{\infty} \frac{j_{k}\left(E_{k}\right) E_{k}}{v_{k}\left(E_{k}\right)} \mathrm{d} E_{k}
$$

where $j_{k}\left(E_{k}\right)$ is the particle's LIS spectrum and $v_{k}\left(E_{k}\right)=c\left(E_{k}^{2} / m_{k}^{2} c^{4}+\right.$ $\left.2 E_{k} / m_{k} c^{2}\right)^{1 / 2} /\left(1+E_{k} / m_{k} c^{2}\right)$ is the velocity of particle $k$ with kinetic energy $E_{k}$. We compute the total energy density of $\mathrm{CR}$ as $\sum_{k} \mathcal{E}_{k} \approx(1+\xi) \mathcal{E}_{p}$, where $\xi=0.41$ is the correction factor for the abundance of He and heavy nuclei. We found that the total CR energy density varies from a minimum of $0.97 \mathrm{eV} \mathrm{cm}^{-3}$ (W98 plus C00) and a maximum of $1.80 \mathrm{eV} \mathrm{cm}^{-3}$ (M02 plus E00), corresponding to an equipartition magnetic field of $6.2 \mu \mathrm{G}$ and $8.5 \mu \mathrm{G}$, respectively. These equipartition values are compatible with the "standard" value of the magnetic field of $6.0 \pm 1.8 \mu \mathrm{G}$ in the cold neutral medium of the Galaxy (Heiles \& Troland [28]).

Table 2 CR ionisation rates $\zeta_{k}^{\mathrm{H}_{2}}$ and $\zeta_{k}^{\mathrm{He}}$, electron production rate $\zeta_{k}^{e}$, energy densities $\mathcal{E}_{k}$, and exponent of the power-law extrapolation of the spectrum at low energies for CR protons ${ }^{a}(p)$ and electrons $(e)$.

\begin{tabular}{lllllll}
\hline$k$ & ref. & $\begin{array}{l}\zeta_{k}^{\mathrm{H}_{2}} \\
{\left[\mathrm{~s}^{-1}\right]}\end{array}$ & $\begin{array}{l}\zeta_{k}^{\mathrm{He}} \\
{\left[\mathrm{s}^{-1}\right]}\end{array}$ & $\begin{array}{l}\zeta_{k}^{e} \\
{\left[\mathrm{~s}^{-1}\right]}\end{array}$ & $\begin{array}{l}\mathcal{E}_{k} \\
{\left[\mathrm{eV} \mathrm{cm}^{-3}\right]}\end{array}$ & $\beta$ \\
\hline \hline$p$ & $\mathrm{~W} 98$ & $2.08 \times 10^{-17}$ & $1.33 \times 10^{-17}$ & $2.50 \times 10^{-17}$ & 0.953 & 0.95 \\
$p$ & M02 & $1.48 \times 10^{-14}$ & $2.16 \times 10^{-15}$ & $3.49 \times 10^{-15}$ & 1.23 & -1 \\
\hline$e$ & $\mathrm{C} 00$ & $1.62 \times 10^{-19}$ & $1.05 \times 10^{-19}$ & $1.94 \times 10^{-19}$ & 0.0167 & 0.08 \\
$e$ & E00 & $6.53 \times 10^{-12}$ & $2.46 \times 10^{-12}$ & $7.45 \times 10^{-12}$ & 0.571 & -1 \\
\hline
\end{tabular}

${ }^{a}$ The proton ionisation rates include the contribution of heavy nuclei.

\section{Energy losses of CRs in the ISM}

The penetration of primary CR and secondary particles in interstellar clouds was studied by Takayanagi [54] and in more detail by Umebayashi \& Nakano [56]. The quantity which describes the "degradation spectrum" of the CR component $k$ resulting from the energy loss of the incident particles is called energy loss function, defined by 


$$
L_{k}\left(E_{k}\right)=-\frac{1}{n\left(\mathrm{H}_{2}\right)}\left(\frac{\mathrm{d} E_{k}}{\mathrm{~d} \ell}\right)
$$

where $n\left(\mathrm{H}_{2}\right)$ is the density of the medium in which the particles propagate and $\ell$ is the path length. Since we consider only energy losses in collisions with $\mathrm{H}_{2}$, our results are applicable only to clouds in which hydrogen is mostly in molecular form. Some energy loss processes are common to CR protons and electrons, like Coulomb interactions, inelastic collisions and ionisation; others are peculiar to protons (elastic collisions, pion production and spallation), others to electrons (bremßtrahlung, synchrotron emission and inverse Compton scattering). These processes are briefly reviewed in the following subsections.

\subsection{Energy loss of protons colliding with $\mathrm{H}_{2}$}

To determine the energy loss function of protons we have used the results collected by Phelps 44 for energies in the range from $10^{-1} \mathrm{eV}$ to $10^{4} \mathrm{eV}$. For higher energies, between $1 \mathrm{keV}$ and $10 \mathrm{GeV}$, we have used data from the NIST Databas 1 for atomic hydrogen multiplied by a factor of 2 to obtain the corresponding values for collisions with molecular hydrogen. NIST data do not include pion production at energies higher than about $0.5 \mathrm{GeV}$, that we computed following Schlickeiser [49]. The resulting energy loss function is shown in the left panel of Fig. 3. The broad peak in $L_{p}\left(E_{p}\right)$ at $E_{p} \approx 10 \mathrm{eV}$ is due to elastic collisions and to the excitation of rotational and vibrational levels, the peak at $E_{p} \approx 100 \mathrm{keV}$ to ionisation, and the rapid increase at energies above $\sim 1 \mathrm{GeV}$ is due to pion production. For the low ionisation levels characteristic of molecular clouds, the energy loss for Coulomb interactions of CRs with ambient electrons can be neglected at energies above $\sim 1 \mathrm{eV}$ (dashed line in the left panel of Fig. 3), since $L_{p}\left(E_{p}\right) \propto n_{e} E_{p}^{-0.5}$, where $n_{e}$ is the electron density of the traversed matter [49].

\subsection{Energy loss of electrons colliding with $\mathrm{H}_{2}$}

To determine the electron energy loss function we have adopted the results of Dalgarno et al. [13] for $10^{-2} \mathrm{eV} \leq E_{e} \leq 1 \mathrm{keV}$ and those of Cravens, Victor \& Dalgarno [11] for $1 \mathrm{eV} \leq E_{e} \leq 10 \mathrm{keV}$. For higher energies, $10 \mathrm{keV} \leq$ $E_{e} \leq 10 \mathrm{GeV}$, we have adopted the loss function for electron-H collisions from the NIST Database multiplied by a factor of 2 . The resulting energy loss function is also shown in the left panel of Fig. 3. The first peak in $L_{e}\left(E_{e}\right)$ is due to the excitation of vibrational levels, the second to the excitation of

${ }^{1}$ http://physics.nist.gov/PhysRefData/Star/Text 
the electronic levels and ionisation, while at higher energies the energy loss function is dominated by bremßtrahlung. As in the case of CR protons, we can neglect the contribution of Coulomb interactions for electrons at energies above $\sim 1 \mathrm{eV}$, since $L_{e}\left(E_{e}\right) \propto n_{e}^{0.97} E_{p}^{-0.44}[51$.
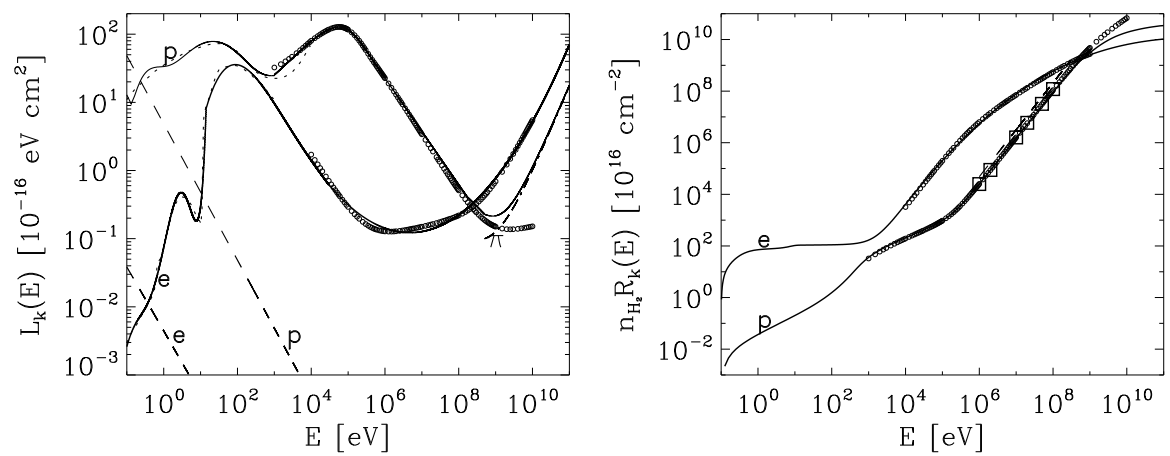

Fig. 3 Left panel: energy loss functions $L_{e}\left(E_{e}\right)$ and $L_{p}\left(E_{p}\right)$ for electrons and protons, respectively, colliding with $\mathrm{H}_{2}$ (solid curves), compared with NIST data (circles); dashed curves show Coulomb losses for a fractional electron abundance $n_{e} / n\left(\mathrm{H}_{2}\right)=10^{-7}$; dash-dotted curve labeled with $\pi$ represents the energy loss by pion production computed following Schlickeiser [49]; dotted curves show the results by Phelps [4] and Dalgarno et al. [13] for $p-\mathrm{H}_{2}$ and $e-\mathrm{H}_{2}$, respectively. Right panel: range $R_{e}\left(E_{e}\right)$ and $R_{p}\left(E_{p}\right)$ for electrons and protons colliding with $\mathrm{H}_{2}$ (solid curves), respectively, compared with NIST data (circles) and the results of Cravens \& Dalgarno [12], squares; the dashed curve shows the fit by Takayanagi [54].

\section{Propagation of a cosmic ray in a molecular cloud}

We assume a plane-parallel geometry and we follow the propagation of CR particles inside a molecular cloud with the so-called continuous-slowing-down approximation (hereafter CSDA) which is also referred as the continuous energy loss regime or thick target approximation when the propagation is dominated by these losses [47, 48. It is useful to introduce the column density of molecular hydrogen $N\left(\mathrm{H}_{2}\right)$,

$$
N\left(\mathrm{H}_{2}\right)=\int n\left(\mathrm{H}_{2}\right) \mathrm{d} \ell,
$$

and to rewrite the energy loss function (Eq. 8) as

$$
L_{k}\left(E_{k}\right)=-\frac{\mathrm{d} E_{k}}{\mathrm{~d} N\left(\mathrm{H}_{2}\right)} .
$$


Let us then define $j_{k}\left(E_{k}, N\right)$ as the spectrum of CR particles of species $k$ at depth $N\left(\mathrm{H}_{2}\right)$, with $j_{k}\left(E_{k}, 0\right)$ representing the LIS spectrum incident on the cloud's surface, defined by a column density $N\left(\mathrm{H}_{2}\right)=0$. To compute $j_{k}\left(E_{k}, N\right)$ we must consider all the processes that degrade the energy of the incident CR particles. Assuming that the direction of propagation does not change significantly inside the cloud, it follows from Eq. (10) that particles of initial energy $E_{k, 0}$ reach energy $E_{k}<E_{k, 0}$ as a consequence of energy losses after propagating across a column density $N\left(\mathrm{H}_{2}\right)$ given by

$$
N\left(\mathrm{H}_{2}\right)=-\int_{E_{k, 0}}^{E_{k}} \frac{\mathrm{d} E_{k}}{L_{k}\left(E_{k}\right)}=n\left(\mathrm{H}_{2}\right)\left[R_{k}\left(E_{k, 0}\right)-R_{k}\left(E_{k}\right)\right],
$$

where $R_{k}\left(E_{k}\right)$ is the range, defined as

$$
R_{k}\left(E_{k}\right)=\int_{E_{k}}^{0} \mathrm{~d} \ell=\int_{0}^{E_{k}} \frac{\mathrm{d} E_{k}}{-\left(\mathrm{d} E_{k} / \mathrm{d} \ell\right)}=\frac{1}{n\left(\mathrm{H}_{2}\right)} \int_{0}^{E_{k}} \frac{\mathrm{d} E_{k}}{L_{k}\left(E_{k}\right)} .
$$

In the right panel of Fig. 3 we show the quantity $n\left(\mathrm{H}_{2}\right) R_{k}\left(E_{k}\right)$ for $k=p, e$, obtained with a numerical integration of Eq. (12), compared with data from the NIST Database at energies from $1 \mathrm{keV}$ to $10 \mathrm{GeV}$ for protons and from $10 \mathrm{keV}$ to $1 \mathrm{GeV}$ for electrons. For protons we also show the fit adopted by Takayanagi [54 in a limited range of energies and the results of Cravens \& Dalgarno [12]. As one can see, except for energies higher than $\sim 100 \mathrm{MeV}$, where the NIST data do not include energy losses by pion production, there is a complete agreement between our results and the NIST data.

Conservation of the number of CR particles of each species implies

$$
j_{k}\left(E_{k}, N\right) \mathrm{d} E_{k}=j\left(E_{k, 0}, 0\right) \mathrm{d} E_{k, 0},
$$

where, for a given value of $N\left(\mathrm{H}_{2}\right)$, the infinitesimal variation $\mathrm{d} E_{k, 0}$ of the particle's initial energy corresponds to an infinitesimal variation $\mathrm{d} E_{k}$ of its energy at a depth $N\left(\mathrm{H}_{2}\right)$ given by

$$
\frac{\mathrm{d} E_{k}}{L_{k}\left(E_{k}\right)}=\frac{\mathrm{d} E_{k, 0}}{L_{k}\left(E_{k, 0}\right)}
$$

We ignore here that electron capture reactions of CR protons with $\mathrm{H}_{2}$ and He do not conserve the number of $\mathrm{CR}$ protons and also the $\alpha+\alpha$ fusion reactions that form ${ }^{6} \mathrm{Li}$ and ${ }^{7} \mathrm{Li}$ because of the small cross sections (Meneguzzi et al. [35]). Thus, the relation between the incident spectrum $j_{k}\left(E_{k, 0}, 0\right)$ and the spectrum $j_{k}\left(E_{k}, N\right)$ at depth $N\left(\mathrm{H}_{2}\right)$ in the CSDA is

$$
j_{k}\left(E_{k}, N\right)=j_{k}\left(E_{k, 0}, 0\right) \frac{\mathrm{d} E_{k}}{\mathrm{~d} E_{k, 0}}=j_{k}\left(E_{k, 0}, 0\right) \frac{L_{k}\left(E_{k, 0}\right)}{L_{k}\left(E_{k}\right)} .
$$




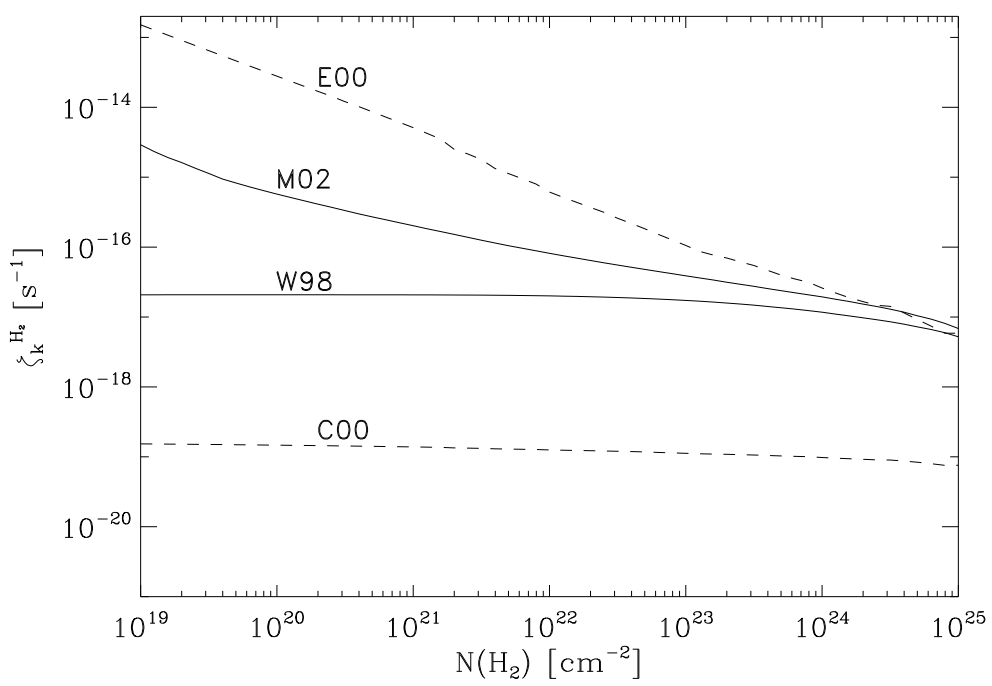

Fig. $4 \mathrm{CR}$ ionisation rate $\zeta_{k}^{\mathrm{H}_{2}}$ as a function of the column density $N\left(\mathrm{H}_{2}\right)$. Solid curves, contribution of CR protons (spectra W98 and M02); dashed curves, contribution of CR electrons (spectra C00 and E00).

We are now able to calculate the $\mathrm{CR}$ ionisation rate inside a molecular cloud as a function of the column density, with the attenuated spectra given by Eq. (15). We compute the CR ionisation rate for $N\left(\mathrm{H}_{2}\right)$ between $10^{19} \mathrm{~cm}^{-2}$ and $10^{25} \mathrm{~cm}^{-2}$, and we show the results for the four incident LIS spectra in Fig. (4) Since we assume an isotropic CR distribution (see Sect. 2), the ionisation rate calculated for a given $N\left(\mathrm{H}_{2}\right)$ corresponds to the ionisation rate at the centre of a spherical cloud with radius $N\left(\mathrm{H}_{2}\right)$.

As a result of the detailed treatment of CR propagation, the decrease of the ionisation rate with increasing penetration in the cloud at column densities in the range $\sim 10^{20}-10^{25} \mathrm{~cm}^{-2}$ is characterised by a power-law behaviour, rather than exponential attenuation, and can be approximated as

$$
\zeta_{k}^{\mathrm{H}_{2}} \approx \zeta_{0, k}\left[\frac{N\left(\mathrm{H}_{2}\right)}{10^{20} \mathrm{~cm}^{-2}}\right]^{-a} .
$$

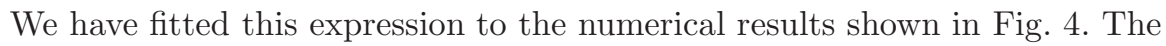
coefficients $\zeta_{0, k}$ and $a$ are given in Table 3. The exponential attenuation of the CR ionisation rate sets in for column densities larger than $\sim 10^{25} \mathrm{~cm}^{-2}$, where $\zeta_{k}^{\mathrm{H}_{2}}$ depends essentially on the flux of CR particles in the high-energy tail of the incident spectrum (above $\sim 0.1-1 \mathrm{GeV}$ ), and directly measurable on the Earth. 
Table 3 Fitting coefficients for Eq. (16) for CR protons ( $p$, also including heavy nuclei) and electrons (e).

\begin{tabular}{lll}
\hline$k$ spectrum $\zeta_{0, k}$ & $a$ \\
& {$\left[\mathrm{~s}^{-1}\right]$} & \\
\hline \hline$p$ W98 & $2.0 \times 10^{-17}$ & 0.021 \\
$p$ M02 & $6.8 \times 10^{-16}$ & 0.423 \\
\hline$e$ C00 & $1.4 \times 10^{-19}$ & 0.040 \\
$e$ E00 & $2.6 \times 10^{-14}$ & 0.805 \\
\hline
\end{tabular}

It is important to stress that a large contribution to the ionisation of $\mathrm{H}_{2}$ comes from low-energy protons and electrons constantly produced (in our steady-state model) by the slowing-down of more energetic particles loosing energy by interaction with the ambient $\mathrm{H}_{2}$. In Fig. 5 we show the differential contribution of $\mathrm{CR}$ protons and electrons to the ionisation rate at a depth of $N\left(\mathrm{H}_{2}\right)=10^{22} \mathrm{~cm}^{-2}$, corresponding to the typical column density of a dense cloud. For protons and heavy nuclei, the bulk of the ionisation is provided by $\mathrm{CR}$ in the range $1 \mathrm{MeV}-1 \mathrm{GeV}$ and by a "shoulder" in the range 1$100 \mathrm{keV}$ produced by slowed-down protons while, for electrons, the largest contribution to the ionisation is distributed over energies in the range $10 \mathrm{keV}-$ $10 \mathrm{MeV}$. This low-energy tail is produced during the propagation of $\mathrm{CR}$ protons and electrons in the cloud even when the incident spectrum is devoid of low-energy particles. Thus, the ionisation rate at any depth in a cloud cannot be calculated by simply removing from the incident spectrum particles with energies corresponding to ranges below the assumed depth.

\section{Comparison with observations}

To obtain the total CR ionisation rate in molecular clouds, we sum the ionisation rates of protons (corrected for heavy nuclei) and electrons. With two possible spectra for each component, we obtain four possible profiles of $\zeta^{\mathrm{H}_{2}}$. These are shown in Fig. 6 as a function of $N\left(\mathrm{H}_{2}\right)$, compared with a compilation of empirical determinations of $\zeta^{\mathrm{H}_{2}}$ in diffuse and dense environments.

The comparison between model results and observational data shown in Fig. 6 should be taken as indicative and interpreted in a statistical sense, as also suggested by the large spread of values of $\zeta^{\mathrm{H}_{2}}$ at each value of $N\left(\mathrm{H}_{2}\right)$. First, the observational $N\left(\mathrm{H}_{2}\right)$ is the entire column density through the cloud, whereas the model $N\left(\mathrm{H}_{2}\right)$ is the column traversed by CRs incident over the cloud's surface. The exact relation between the quantities depend on factors like the cloud geometry and orientation with respect to the line-of-sight, and the variation of $\mathrm{CR}$ ionisation rate with depth within the cloud. In addition, for the cloud cores of Caselli et al. [5 we adopted the $\mathrm{H}_{2}$ column density estimated by Butner et al. [3] from measurements of $\mathrm{C}^{18} \mathrm{O}$ multiplied by a factor 


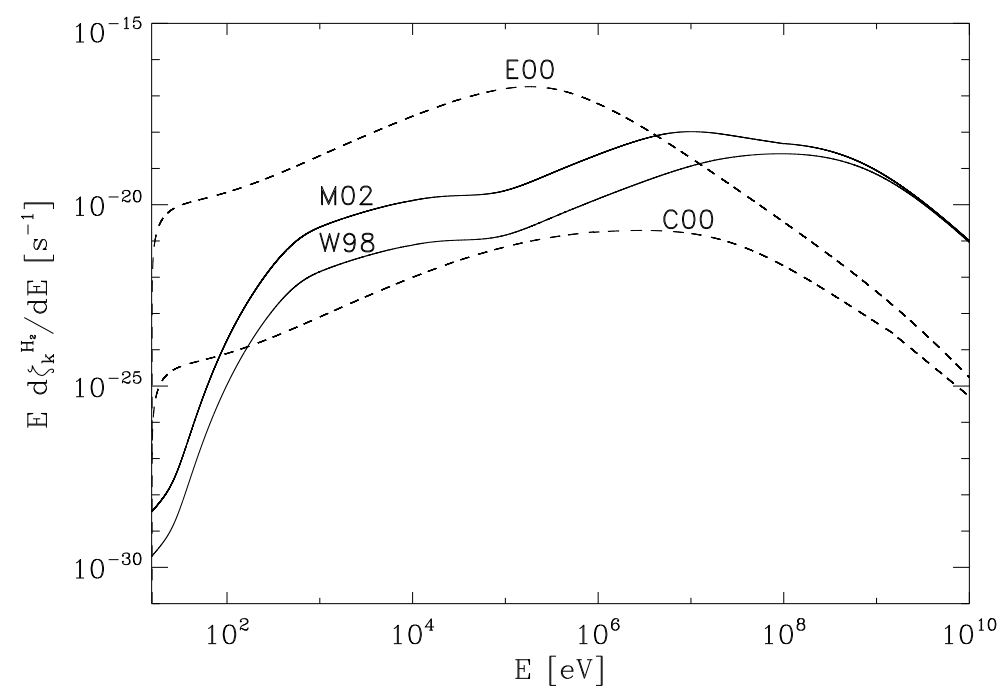

Fig. 5 Differential contribution to the ionisation rate $E \mathrm{~d} \zeta_{k}^{\mathrm{H}_{2}} / \mathrm{d} E$ per logarithmic interval of kinetic energy, for the four spectra considered at a depth $N\left(\mathrm{H}_{2}\right)=10^{22} \mathrm{~cm}^{-2}$ (solid curves, protons; dashed curves, electrons).

of 2, to account for depletion of CO onto grains (Caselli et al. [5]). Second, many of the sight-lines where $\zeta^{\mathrm{H}_{2}}$ has been determined in diffuse clouds may have multiple cloud components, which would reduce the column density of a single cloud. It is probably safe to conclude that the observational column density is an upper limit to the column density traversed by CRs incident on each cloud, and therefore the data shown in Fig. [6]should probably be shifted to the left by a factor of 2 or so. At any rate, from the comparison with observational data, shown in Fig. [6] we can draw the following conclusions:

1. Although the gas column density of the object is by no means the only parameter controlling the $\mathrm{CR}$ ionisation rate, the data suggest a decreasing trend of $\zeta^{\mathrm{H}_{2}}$ with increasing $N\left(\mathrm{H}_{2}\right)$, compatible with our models $\mathrm{M} 02+\mathrm{C} 00, \mathrm{~W} 98+\mathrm{E} 00, \mathrm{~W} 98+\mathrm{C} 00$. However, the measured values of $\zeta^{\mathrm{H}_{2}}$ are very uncertain, especially in dense environments. Part of the large spread in the sample of cloud cores may be due to a poor understanding of the chemistry.

2. The highest values of $\zeta^{\mathrm{H}_{2}}$, measured in diffuse clouds sight lines, could be explained if $\mathrm{CR}$ electrons are characterised by a rising spectrum with decreasing energy. The E00 spectrum represents an extreme example of this kind, and it results in values of $\zeta^{\mathrm{H}_{2}}$ somewhat in excess of the diffuse clouds observations. The same spectrum accounts simultaneously for the $\mathrm{CR}$ ionisation rates measured in most protostellar envelopes of much 


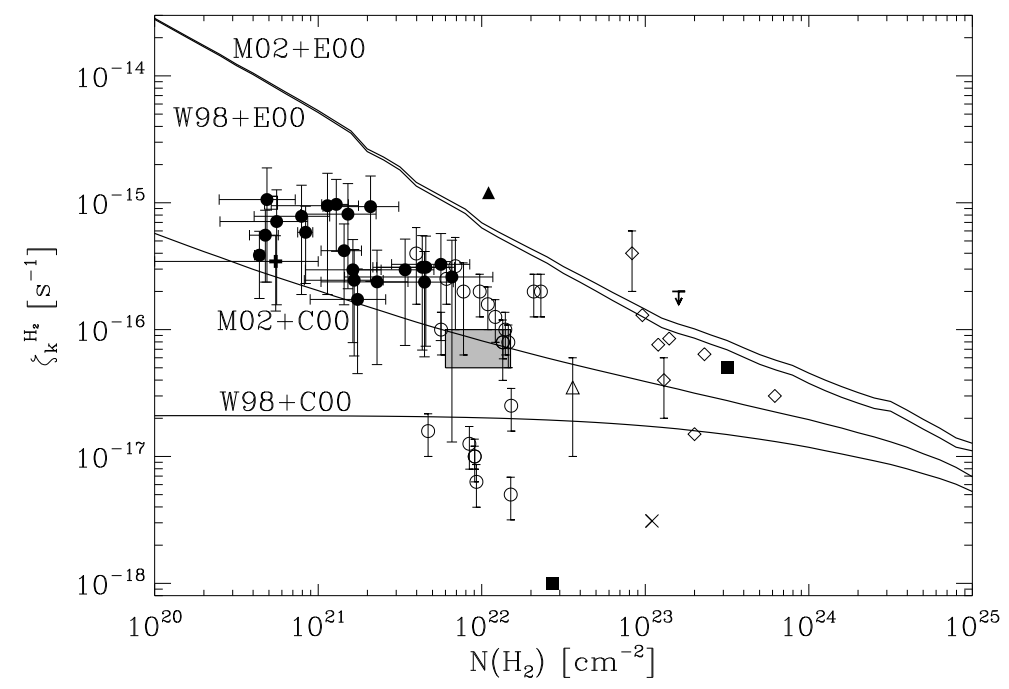

Fig. 6 Total CR ionisation rate $\zeta^{\mathrm{H}_{2}}$ as a function of $N\left(\mathrm{H}_{2}\right)$ according to our models ( solid curves). Observational data: filled circles, diffuse clouds (Indriolo et al. [32]); empty square, diffuse cloud W49N (Neufeld et al. [40); empty circles, dense cores (Caselli et al. 51); empty triangle, prestellar core B68 (Maret \& Bergin 34); filled squares, T Tauri disks TW Hya and DM Tau (Ceccarelli et al. [6]); filled triangle, SNR W51C (Ceccarelli et al. 7]); diamonds, protostellar envelopes (de Boisanger, Helmich, \& van Dishoeck [14, van der Tak et al. [58, van der Tak \& van Dishoeck [57], Doty et al. [16, and Hezareh et al. 29]); cross, massive star-forming region DR21(OH) (Hezareh et al. 29]). The filled box indicates the range of column densities and CR ionisation rates compatible with the data analysed by Williams et al. 61.

higher column density. Conversely, a spectrum of protons and heavy nuclei rising with decreasing energy, like the M02 spectrum, can provide alone a reasonable lower limit for the $\mathrm{CR}$ ionisation rate measured in diffuse clouds.

3. Without a significant low-energy (below $\sim 100 \mathrm{MeV}$ ) component of electrons and/or protons and heavy nuclei, it is impossible to reproduce the large majority of observations. The combination of the C00 spectrum for electrons with the W98 spectrum for protons and heavy nuclei clearly fails over the entire range of column densities.

\section{Effects of magnetic field on CR propagation}

The high values of $\zeta^{\mathrm{H}_{2}}$ in the diffuse interstellar gas can be reconciled with the lower values measured in cloud cores and massive protostellar envelopes 
by invoking various mechanisms of $\mathrm{CR}$ screening in molecular clouds due to either self-generated Alfvén waves in the plasma [50, 26, 41] or to focusing and magnetic mirror effects 9, 10, 43. Besides, a few molecular cloud cores and one dense envelope (Fig. 6) are characterised by $\zeta^{\mathrm{H}_{2}} \leq 10^{-17} \mathrm{~s}^{-1}$ and probably can only be explained by invoking the CR suppression mechanisms. Mirroring and focusing are due to the non-uniformity of the large-scale (mean) component of the field, whereas diffusion is associated to magnetic field fluctuations on the scale of the Larmor radius of CR particles. The relative importance of these processes in the ISM depends on a number of variables not always well determined (geometry and strength of the magnetic field, nature and characteristics of turbulence, etc.), and is unclear whether they can significantly reduce (or enhance) the CR ionisation of molecular cloud cores. In addition, the damping of small-scale magnetic fluctuations (e.g. Alfvén waves) that affect the propagation of CRs is strongly dependent on the ionisation fraction of the medium, which, in turn, is mostly determined by the CRs themselves.

CRs perform an helicoidal motion around the magnetic field lines. For a uniform magnetic field $B=10 \mu \mathrm{G}$, the Larmor radii, $r_{\mathrm{L}}$ of ionising CRs (CR protons and heavy nuclei with $E \lesssim 1 \mathrm{GeV} /$ nucleon and $\mathrm{CR}$ electrons with $E \lesssim 10 \mathrm{MeV}$, see Padovani et al. [42]) are less than $\sim 10^{-7} \mathrm{pc}$ and $\sim 10^{-9} \mathrm{pc}$ for protons and electrons, respectively, many orders of magnitude smaller than the typical size of Bok globules, dense cores, and giant molecular clouds [43, see Fig. (7) In the absence of small-scale perturbations in the field, we can therefore assume that low-energy CRs propagate closely following the magnetic field lines.

Perturbations in the forms of magnetohydrodynamic (MHD) waves with wavelength of the order of the Larmor radius of the particle can efficiently scatter CRs. The waves can be part of an MHD turbulent cascade, or can be self-generated by the CRs themselves [30. However, in a mostly neutral ISM, turbulent MHD cascades are quenched at scales of roughly the collision mean free path of ions with neutrals, if the ion-neutral collision rate exceeds the energy injection rate. Alfvén waves are efficiently damped by collision with neutrals below a critical wavelength $\lambda_{\text {cr }}$ 31] and, for typical molecular cloud conditions, $\lambda_{\mathrm{cr}} \approx 3 \times 10^{-3} \mathrm{pc}[45$. Thus, for typical values of the cloud's parameters, only $\mathrm{CR}$ particles with energy larger than a few $\mathrm{TeV}$, and Larmor radii $r_{\mathrm{L}} \geq \lambda_{\text {cr }}$ find MHD waves to resonate with. These particles however do not contribute significantly to the ionisation of the cloud.

We expect that CRs in the energy range between $100 \mathrm{MeV} /$ nucleon and $1 \mathrm{GeV} /$ nucleon, that provide the bulk of the ionisation in a cloud core, stream freely through the core without self-generating MHD waves. We ignore therefore the presence of self-generated waves in cloud cores in the following, but we believe that this problem deserves further scrutiny. 


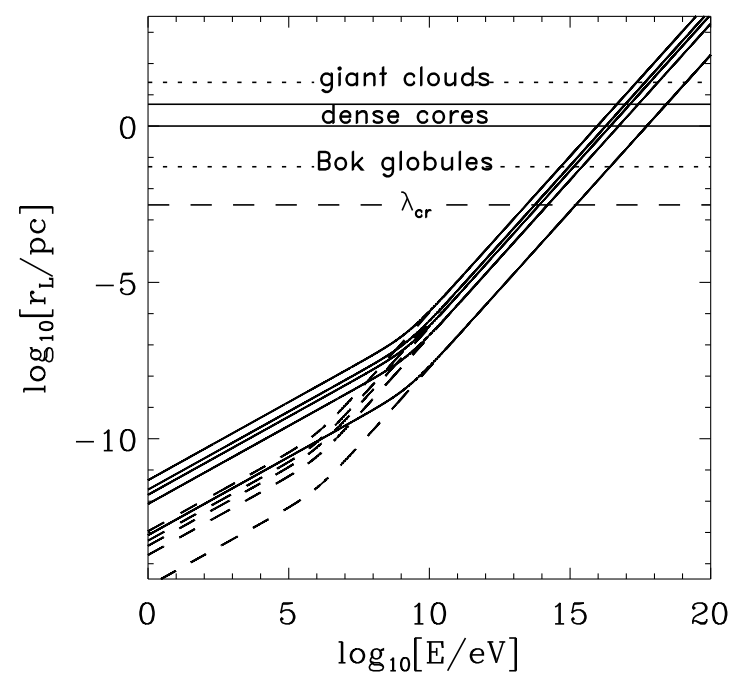

Fig. 7 Larmor radius as a function of the cosmic-ray energy for $\alpha=$ 1, 10, 20,30, and 90. Solid and dashed lines represent cosmic-ray protons and electrons, respectively.

\section{Magnetic focusing and mirroring}

Theoretical models predict that collapsing cloud cores must overcome the support provided by their magnetic field in order to form stars. In the process, the competition between gravity pulling inward and magnetic pressure pushing outward is expected to produce a warped, hourglass pattern of the magnetic field. Recently, this scenario has received support from observations [22, 55] showing a magnetic field geometry consistent with the formation of solar-type stars, in which ordered large-scale magnetic fields control the evolution and collapse of molecular cloud cores 24. We therefore adopt the hourglass geometry as the basis of our analysis of CR penetration into a cloud core.

The effects of magnetic mirroring and focusing in a hourglass geometry can be simply described following e.g. Desch et al. 15. A charged particle travelling in a magnetised medium conserves its kinetic energy $\gamma m c^{2}$ and its magnetic moment $\mu=\gamma m v^{2} \sin ^{2} \alpha / 2 B$. It follows that CRs propagating from the intercloud medium (ICM) to the cloud's interior must increase $v_{\perp}^{2}$ to conserve $\mu$ and decrease $v_{\|}$to conserve $|\mathbf{v}|^{2}$. Thus, the pitch angle of the particle must increase from the value $\alpha_{\mathrm{ICM}}$ to a value $\alpha$ as

$$
\frac{\sin ^{2} \alpha}{\sin ^{2} \alpha_{\mathrm{ICM}}}=\frac{B}{B_{\mathrm{ICM}}} \equiv \chi
$$


where $\chi>1$. Therefore, a CR starting in the ICM with a pitch angle $\sin \alpha_{\mathrm{ICM}}>1 / \chi^{1 / 2}$ cannot penetrate a region with magnetic field $B>\chi B_{\mathrm{ICM}}$, and will be bounced out (magnetic mirroring). Conversely, the CR flux $j(E)$ in the cloud is increased by the opening out of the field lines by a factor proportional to the density of magnetic field lines per unit area (magnetic focusing),

$$
j(E)=\chi j_{\text {ICM }}(E) .
$$

The effects of focusing and mirroring depend only on the magnetic field strength, and are the same for CR protons, electrons, and heavy nuclei.

\section{$9 \mathrm{CR}$ ionisation rate in presence of a magnetic field}

In order to study the effects of the magnetic field on the propagation of CRs in molecular cores, we adopt the models of Li \& Shu [33] (see also Galli et al. [19), for magnetostatic, scale-free, self-gravitating clouds supported by axially-symmetric hourglass-like magnetic fields. For simplicity, we consider models with an isothermal equation of state. These models are characterised by a value of the non-dimensional mass-to-flux ratio $\lambda$ defined by

$$
\lambda=2 \pi G^{1 / 2} \frac{M(\Phi)}{\Phi},
$$

where $G$ is the gravitational constant, $\Phi$ the magnetic flux, and $M(\Phi)$ the mass contained in the flux tube $\Phi$. For our reference model we choose $\lambda=$ 2.66 , assuming a sound speed $c_{\mathrm{s}}=0.2 \mathrm{~km} \mathrm{~s}^{-1}$ and an intercloud magnetic field $B_{\mathrm{ICM}}=3 \mu \mathrm{G}$. We focus on a flux tube enclosing a mass $M(\Phi)=1 \mathrm{M}_{\odot}$, a typical value for a low-mass core. The following equations are written as a function of the polar angle $\theta$ : a $\mathrm{CR}$ is outside the core for $\theta=0$ and reaches the midplane when $\theta=\pi / 2$.

Following the assumptions in Sect. 8. CRs starting with pitch angles $\alpha_{\mathrm{ICM}}$ smaller than a critical value $\alpha_{\mathrm{cr}}$ are able to reach the cloud's midplane. When $\alpha_{\mathrm{ICM}}>\alpha_{\mathrm{cr}}$ then CRs will be pushed back by magnetic mirroring before reaching the midplane at a position $\theta_{\max }\left(\alpha_{\mathrm{ICM}}\right)<\pi / 2$. Inverting this relation, one finds the value of the maximum allowed pitch angle $\alpha_{\mathrm{ICM} \text { max }}(\theta)$ for a $\mathrm{CR}$ to reach a given position $\theta$. Figure 8 shows the variation of the pitch angle computed from Eq. (17) assuming the magnetic field profile of the reference $1 \mathrm{M}_{\odot}$ flux tube. is

The contribution to the $\mathrm{CR}$ ionisation rate of $\mathrm{H}_{2}$ for $\mathrm{CRs}$ entering the core

$$
\begin{aligned}
\zeta^{\mathrm{H}_{2}}(\theta)= & 2 \pi \chi(\theta) \int_{0}^{\infty} \mathrm{d} E \int_{0}^{\alpha_{\mathrm{ICM}, \max }(\theta)} j\left[E, N\left(\theta, \alpha_{\mathrm{ICM}}\right)\right] \\
& \times[1+\phi(E)] \sigma^{\mathrm{ion}}(E) \sin \alpha_{\mathrm{ICM}} \mathrm{d} \alpha_{\mathrm{ICM}},
\end{aligned}
$$




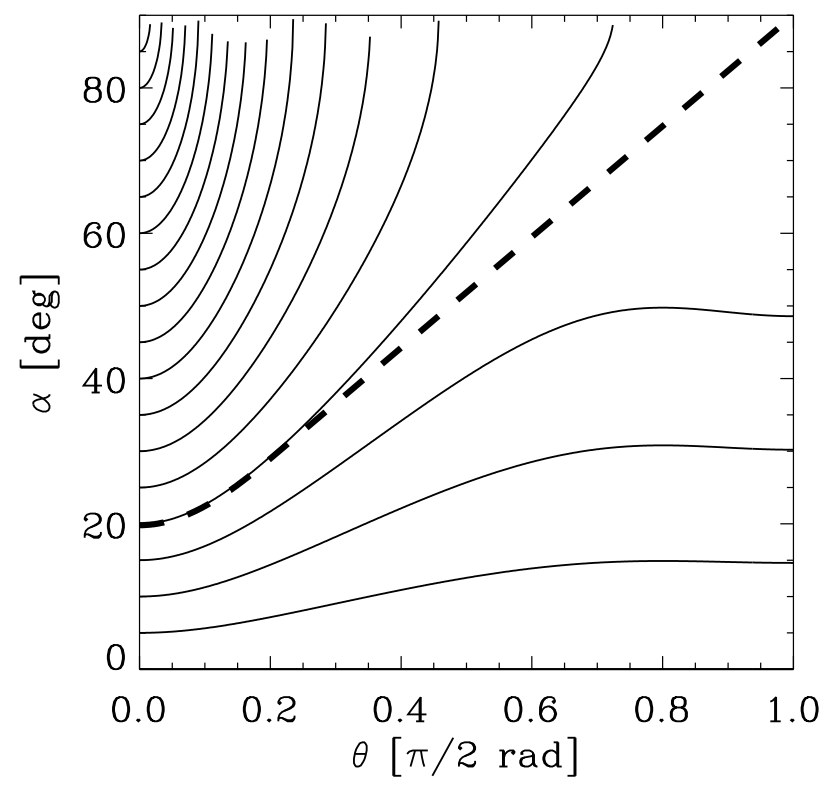

Fig. 8 Variation of the CR pitch angle as a function of the polar angle for values of $\alpha_{\mathrm{ICM}}$ between $0^{\circ}$ and $90^{\circ}$ in steps of $5^{\circ}$. The dashed line represents the critical pitch angle $\alpha_{\mathrm{cr}}=20.5^{\circ}$.

where $N\left(\theta, \alpha_{\mathrm{ICM}}\right)$ is the column density of $\mathrm{H}_{2}$ into the core (with $N=0$ at $\theta=0), \phi(E)$ is given by Eq. (4), $\sigma^{\text {ion }}(E)$ is the ionisation cross section of $\mathrm{H}_{2}$ (see Sect. 2), and the column density passed through by a CR propagating along a magnetic field line is

$$
N\left(\theta, \alpha_{\mathrm{ICM}}\right)=\frac{1}{\mu m_{\mathrm{H}}} \int \rho \frac{\mathrm{d} \ell}{\cos \alpha},
$$

where $\mu=2.8$ is the molecular weight, $\mathrm{d} \ell=\left[\mathrm{d} r^{2}+(r \mathrm{~d} \theta)^{2}\right]^{1 / 2}$ is the element of magnetic field line, and the factor $1 / \cos \alpha$ accounts for the increase of the actual path length of a CR performing a helicoidal trajectory around a magnetic field with respect to the displacement along the field line. Fig. 9 shows the column density passed through by the CR before reaching the mirror point. As a first approximation one can assume that CRs coming from the ICM and travelling towards the cloud's midplane experience a similar increase in column density, independently on the initial pitch angle $\alpha_{\mathrm{ICM}}$, the latter mainly determining the value of the column density at which the CRs are pushed out by magnetic mirroring. For this reason, we assume for all CRs the column density profile corresponding to $\alpha_{\mathrm{ICM}}=0$, but truncated at increasingly larger values depending on the initial pitch angle $\alpha_{\mathrm{ICM}}$, 


$$
N\left(\theta, \alpha_{\mathrm{ICM}}\right) \approx N(\theta, 0) \quad \text { if } \quad 0<\theta<\theta_{\max }\left(\alpha_{\mathrm{ICM}}\right) .
$$

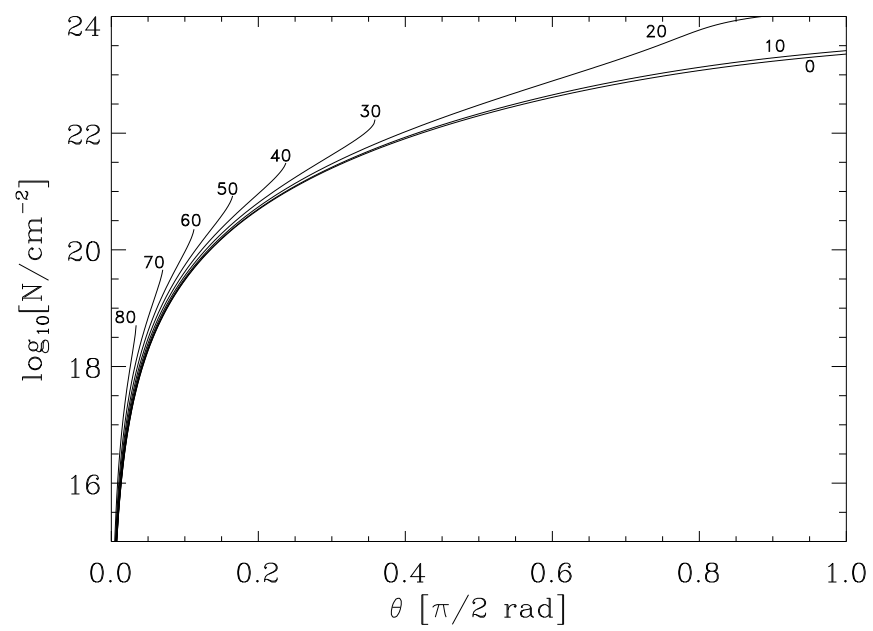

Fig. 9 Column density passed through before reaching the mirror point by CRs propagating along a field line of the reference flux tube enclosing $1 M_{\odot}$, as function of the polar angle $\theta$ for different values of the initial pitch angle $\alpha_{\mathrm{ICM}}$ (labels in degrees).

Adopting the CSDA (see Sect. 55), the total CR ionisation rate, see details in Padovani \& Galli [43, is then given by two contributions due to CRs entering the core from the upper side (subscript + ) and the lower side (subscript -) of the core

$$
\zeta^{\mathrm{H}_{2}}(\theta)=\zeta_{+}^{\mathrm{H}_{2}}(\theta)+\zeta_{-}^{\mathrm{H}_{2}}(\theta),
$$

with

$$
\begin{aligned}
& \zeta_{+}^{\mathrm{H}_{2}}(\theta)=\varphi_{+}(\theta) \zeta_{0}^{\mathrm{H}_{2}}\left[N_{0}(\theta)\right] \\
& \zeta_{-}^{\mathrm{H}_{2}}(\theta)=\varphi_{-}(\theta) \zeta_{0}^{\mathrm{H}_{2}}\left[2 N_{0}(\pi / 2)-N_{0}(\theta)\right] .
\end{aligned}
$$

$\varphi_{+}(\theta)$ and $\varphi_{-}(\theta)$ are factors accounting for magnetic effects

$$
\begin{aligned}
& \varphi_{+}(\theta)=\frac{\chi(\theta)}{2}\left[1-\cos \alpha_{\mathrm{ICM}, \max }(\theta)\right] \\
& \varphi_{-}(\theta)=\frac{\chi(\theta)}{2}\left(1-\cos \alpha_{\mathrm{cr}}\right)
\end{aligned}
$$

and $\zeta_{0}^{\mathrm{H}_{2}}\left[N_{0}(\theta)\right]$ is the $\mathrm{CR}$ ionisation rate in the non-magnetic case, see Eqs. (3), (6), and (5). 
Mirroring and focusing have opposite effects of comparable magnitude on the ionisation rate, both becoming more and more important approaching the core's midplane where the field is stronger. However, as shown in the left panel of Fig. 10, magnetic mirroring always reduces the CR ionisation rate more than magnetic focusing can increase it, the total effect being a net reduction of $\zeta^{\mathrm{H}_{2}}$ by a factor between 2 and 3. Notice that the maximum effect of the magnetic field on $\zeta^{\mathrm{H}_{2}}$ is obtained at an intermediate position corresponding to column densities of $10^{21}-10^{22} \mathrm{~cm}^{-2}$ where the reduction factor $\mathcal{R}=\zeta^{\mathrm{H}_{2}} / \zeta_{0}^{\mathrm{H}_{2}}$ is about 0.3 (see right panel of Fig. 10, while the value at the core's midplane is independent on the assumed CR spectrum, a result also obtained by Desch et al. [15].
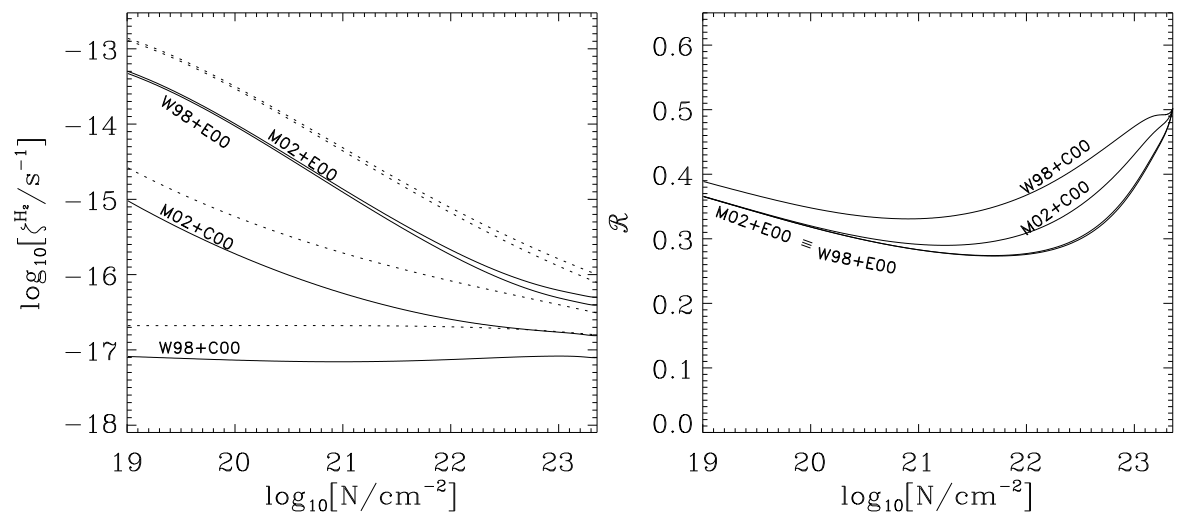

Fig. 10 Left panel: comparison between the CR ionisation rate with and without the effects of magnetic field (solid and dotted lines, respectively) as function of the column density. Right panel: ratio between the ionisation rates in the magnetic and non-magnetic case. The curves are labelled as in Fig. 6]

We explored the parameter space, investigating the variation of the relevant quantities of the problem as a function of the enclosed mass for our reference core. Flux tubes enclosing smaller masses intersect the midplane at smaller equatorial radii $\left(r_{\mathrm{eq}}\right)$ and are characterised by larger values of the magnetic field and density. As a consequence, focusing becomes more important, since $\chi$ increases, but also mirroring becomes more severe, since $\alpha_{\mathrm{cr}}$ decreases. The net effect is a stronger reduction of $\zeta^{\mathrm{H}_{2}}$ in the innermost regions of the core as compared to the envelope (see Table 4). As the field strength increases approaching the central singularity of the model, $\chi \rightarrow \infty$ and the reduction factor of the $\mathrm{CR}$ ionisation rate in the core's midplane approaches the asymptotic value $\mathcal{R}(\pi / 2)=1 / 2$. Conversely, for flux tubes enclosing larger masses, the field strength approaches the ICM value, and the density decreases to zero. Therefore both focusing and mirroring become weaker, and $\alpha_{\mathrm{cr}}$ approaches $90^{\circ}$, as shown by Table 4 As expected, for increasing values of $M(\Phi), \zeta^{\mathrm{H}_{2}}$ approaches the value of the non-magnetic case, 
because the magnetic field strength decreases away from the centre of the core, approaching the ICM value.

Table 4 Values of the parameters described in the text as function of the mass, $M(\Phi)$, contained within flux tube $\Phi$.

\begin{tabular}{cccccc}
\hline \hline $\begin{array}{c}M(\Phi) \\
\left(M_{\odot}\right)\end{array}$ & $\begin{array}{c}r_{\text {eq }} \\
(\mathrm{pc})\end{array}$ & $\begin{array}{c}N_{0}(\pi / 2) \\
\left(10^{23} \mathrm{~cm}^{-2}\right)\end{array}$ & $\alpha_{\mathrm{cr}}$ & $\chi(\pi / 2)$ & $\mathcal{R}(\pi / 2)$ \\
\hline 0.5 & 0.018 & 4.54 & $14.8^{\circ}$ & 15.347 & 0.508 \\
1 & 0.036 & 2.27 & $20.5^{\circ}$ & 8.174 & 0.516 \\
5 & 0.180 & 0.45 & $39.9^{\circ}$ & 2.435 & 0.566 \\
10 & 0.360 & 0.23 & $49.7^{\circ}$ & 1.717 & 0.608 \\
50 & 1.802 & 0.05 & $69.3^{\circ}$ & 1.143 & 0.739 \\
100 & 3.604 & 0.02 & $75.0^{\circ}$ & 1.072 & 0.794 \\
\hline
\end{tabular}

For cores with strong magnetic support (lower values of $\lambda$ ), the equatorial squeezing of the field lines is stronger, and the lines reach more internal regions of the core where the density is higher. As a consequence, $\alpha_{\mathrm{cr}}$ decreases, the mirroring effect becomes stronger, and a smaller fraction of CRs can penetrate the cloud. In Figure 11 we show the reduction factor $\mathcal{R}$ as a function of column density for decreasing values of $\lambda$. For simplicity, we have considered only the combination of the proton spectrum M02 and the electron spectrum C00 (the results obtained with the other spectra are similar). As the Figure shows, the reduction of $\zeta^{\mathrm{H}_{2}}$ is larger in cores with larger magnetic support, due to the increase in the field strength and concentration of field lines. The reduction is a factor $\sim 4$ for the outer regions of cores with $\lambda=1.63$, the lowest value of mass-to-flux ratio considered in our models.

For $\lambda \rightarrow 1$, the density distribution becomes more and more flattened, the core assumes the shape of a thin disk, and the column density from the ICM to the core's midplane becomes larger. For these magnetically dominated disk-like configurations, the reduction of the $\mathrm{CR}$ ionisation rate approaches the asymptotic value $1 / 2$.

\section{Conclusions}

The comparison between our models and the observational data available for diffuse clouds, dense cores and massive protostellar envelopes indicates that good agreement between theory and observations can be obtained for the CR ionisation rate of the ISM by including CR electrons with an energy spectrum increasing towards low energies, as also suggested by Webber 61. Despite the observational uncertainties due to the uncertainty in the CR spectrum at energies below $\sim 1 \mathrm{GeV}$ and the uncertainties in the empirically deter- 


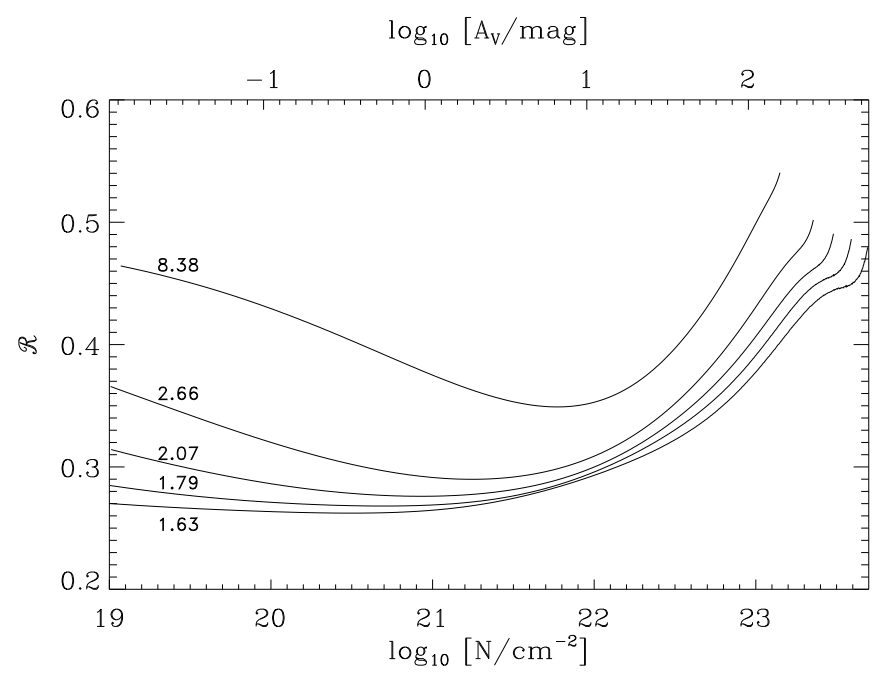

Fig. 11 Reduction factor $\mathcal{R}$ between the $\mathrm{CR}$ ionisation rates in the magnetic and non-magnetic case for the case of M02+C00 spectrum (see Figure 6 for the label). The curves are computed for a flux tube containing $1 M_{\odot}$ and for different values of $\lambda=8.38,2.66,2.07,1.79,1.63$. The upper scale shows the extinction through the cloud obtained from $A_{V} / \mathrm{mag}=N / 10^{21} \mathrm{~cm}^{-2}$.

mined values of $\zeta^{\mathrm{H}_{2}}$ in diffuse and dense molecular clouds, several important conclusions clearly emerge from our study:

1. Values of $\zeta^{\mathrm{H}_{2}}$ measured in diffuse clouds are greater on average by an order of magnitude than those ones measured in dense molecular clouds. If confirmed, these data imply the presence of a $\mathrm{CR}$ proton and/or CR electron spectrum which increases at low energies.

2. Values of $\zeta^{\mathrm{H}_{2}}$ measured in dense molecular clouds span a range of about two orders of magnitude and are subject to considerable uncertainty. It is difficult to establish how much of the observed spread is due to variations in the CR ionisation rate. It is likely that in dense clouds the effects of magnetic fields on the propagation of $\mathrm{CR}$ particles cannot be neglected. In addition, it might be necessary to take into account the density distribution inside each cloud.

3. The values of $\zeta^{\mathrm{H}_{2}}$ measured in massive protostellar envelopes are somewhat higher than the predictions of our models at the corresponding column densities. This seems to suggest the presence of further ionisation sources in these objects, as, for example, X-ray emission from the young stellar objects.

4. $\zeta^{\mathrm{H}_{2}}$ in a magnetised core is always reduced with respect to its non-magnetic value, by a factor depending on the core's mass-to-flux ratio $(\lambda)$ and the amount of mass contained in the flux tube considered. The reduction is 
less severe for flux tubes enclosing larger masses and for larger values of $\lambda$. Thus, the values of $\zeta^{\mathrm{H}_{2}}$ derived for dense cores and globules [5, 62, 34] probably underestimate the "external" (i.e. intercloud) CR ionisation rate by a factor of $\sim 3-4$, thus alleviating the discrepancy with measurements of $\zeta^{\mathrm{H}_{2}}$ in diffuse clouds.

\section{References}

1. Black, J. H. \& Dalgarno, A. 1977, ApJS, 34, 405

2. Black, J. H., Hartquist, T. W. \& Dalgarno, A. 1978, ApJ, 224, 448

3. Butner, H. M., Lada, E. A., Loren, R. B. 1995, ApJ, 448, 207

4. Casadei, D. \& Bindi, V. 2004, ApJ, 612, 262

5. Caselli, P., Walmsley, C. M., Terzieva, R., et al. 1998, ApJ, 499, 234

6. Ceccarelli, C., Dominik, C., Lefloch, B., et al. 2004, ApJ, 607, L51

7. Ceccarelli, C., Hily-Blant, P., Montmerle, T., et al. 2011, ApJL, 740, L4

8. Cecchi-Pestellini, C. \& Aiello, S. 1992, MNRAS, 258, 125

9. Cesarski, C. J. \& Völk, H. J. 1978, A\&A, 70, 367

10. Chandran, B. D. G. 2000, ApJ, 529, 513

11. Cravens, T. E., Victor \& G. A., Dalgarno, A. 1975, Plan. Space Sci., 23, 1059

12. Cravens, T. E. \& Dalgarno, A. 1978, ApJ, 219, 750

13. Dalgarno, A., Yan, M. \& Liu, W. 1999, ApJS, 125, 237

14. de Boisanger, C., Helmich, F. P. \& van Dishoeck, E. F. 1996, ApJ, 463, 181

15. Desch, S. J., Connolly, H. C., Jr. \& Srinivasan, G. 2004, ApJ, 602, 528

16. Doty, S. D., van Dishoeck, E. F., van der Tak, F. F. S., et al. 2002, A\&A, 389, 446

17. Federman, S. R., Weber, J. \& Lambert, D. L. 1996, ApJ, 463, 181

18. Gabici, S., Aharonian, F. A. \& Blasi, P. 2007, Ap\&SS, 309, 365

19. Galli, D., Lizano, S., Li, Z.-Y., et al. 1999, ApJ, 521, 630

20. Geballe, T. R., McCall, B. J., Hinkle, K. H., et al. 1999, ApJ, 510, 251

21. Glassgold, A. E. \& Langer, W. D. 1973, ApJ, 186, 859

22. Girart, J. M., Rao, R. \& Marrone, D. P. 2006, Science, 5788, 812

23. Glassgold, A. E. \& Langer, W. D. 1974, ApJ, 193, 73

24. Gonçalves, J., Galli, D. \& Girart, J. M. 2008, A\&A, 490, L39

25. Hartquist, T. W., Black, J. H. \& Dalgarno, A. 1978, MNRAS, 185, 643

26. Hartquist, T. W., Doyle, H. T. \& Dalgarno, A. 1978, A\&A, 68, 65

27. Hayakawa, S., Nishimura, S. \& Takayanagi, T. 1961, PASJ, 13, 184

28. Heiles, C. \& Troland, T. H. 2005, ApJ, 624, 773 C

29. Hezareh, T., Houde, M., McCoey, C., et al. 2008, ApJ, 684, 1221

30. Kulsrud, R. M. 2005, Plasma physics for astrophysics, Princeton University Press

31. Kulsrud, R. \& Pearce, W. P. 1969, ApJ, 156, 445

32. Indriolo, N. \& McCall, B. J. 2012, ApJ, 745, 91

33. Li, Z.-Y. \& Shu, F. H. 1996, ApJ, 472, 211

34. Maret, S. \& Bergin, E. A. 2007, ApJ, 664, 956

35. Meneguzzi, M., Adouze, J. \& Reeves, H. 1971, A\&A, 15, 377

36. Mewaldt, R. A., Wiedenbeck, M. E., Scott, L. M., et al. 2004, Physics of the Outer Heliosphere, AIP Conference Proceedings, vol. 719, p. 127

37. McCall, B. J., Geballe, T. R., Hinke, K. H., et al. 1998, Science, 279, 1910

38. McCall, B. J., Huneycutt, A. J., Saykally, R. J., et al. 2003, Nature, 422, 500

39. Moskalenko, I. V., Strong, A. W., Ormes, J. F. et al. 2002, ApJ, 565, 280

40. Neufeld, D. A., Sonnentrucker, P., Phillips, T. G., et al. 2010, A\&A, 518, L108

41. Padoan, P. \& Scalo, 2005, ApJ, 624, L97 
42. Padovani, M., Galli, D. \& Glassgold, A. E. 2009, A\&A, 501, 619

43. Padovani, M. \& Galli, D. 2011, A\&A, 503, A109

44. Phelps, A. V. 1990, J. Phys. Chem. Ref. Data, 19, 3

45. Pinto, C., Galli, D. \& Bacciotti, F. 2008, A\&A, 484, 1

46. Potgieter, M. S. 1995, Adv. Space Res., 16 (9), 191

47. Ramaty, R., \& Lingenfelter, R. E. 1975, Solar Gamma-, X-, and EUV Radiation, 68, 363

48. Ramaty, R., Kozlovsky, B., \& Lingenfelter, R. E. 1996, ApJ, 456, 525

49. Schlickeiser, R. 2002, in Cosmic Ray Astrophysics, (Springer: Berlin)

50. Skilling, J. \& Strong, A. W. 1976, A\&A, 53, 253

51. Swartz, W. E., Nisbet, J. S. \& Green, A. E. S. 1971, J. Geophys. Res., 76, 8425

52. Spitzer, L. \& Tomasko, M. G. 1968, ApJ, 152, 971

53. Strong, A. W., Moskalenko, I. V. \& Reimer, O. 2000, ApJ, 537, 763

54. Takayanagi, M. 1973, PASJ, 25, 327

55. Tang, Y.-W., Ho, P. T. P., Koch, P. M., et al. 2009, ApJ, 700, 251

56. Umebayashi, T. \& Nakano, T. 1981, PASJ, 33, 617

57. van der Tak, F. F. S. \& van Dishoeck, E. F. 2000, A\&A, 358, L79

58. van der Tak, F. F. S. \& van Dishoeck, E. F. \& Evans, N. J., II et al. 2000, ApJ, 537,283

59. van Dishoeck, E. F. \& Black, J. H. 1986, ApJS, 62, 109

60. Webber, W. R. 1987, A\&A, 179, 277

61. Webber, W. R. 1998, ApJ, 506, 329

62. Williams, J. P., Bergin, E. A., Caselli, P. et al. 1998, ApJ, 503, 689 